\title{
Generalized MGF of Beckmann Fading with Applications to Wireless Communications Performance Analysis
}

\author{
Juan P. Peña-Martín, Juan M. Romero-Jerez, Senior Member, IEEE, F. J. Lopez-Martinez, Member, IEEE
}

\begin{abstract}
The Beckmann distribution is a general multipath fading model for the received radio signal in the presence of a large number of scatterers, which can thence be modeled as a complex Gaussian random variable where both the inphase and quadrature components have arbitrary mean and variance. However, the complicated nature of this distribution has prevented its widespread use and relatively few analytical results have been reported for this otherwise useful fading model. In this paper, we derive a closed-form expression for the generalized moment-generating function (MGF) of the signal-to-noise ratio (SNR) of Beckmann fading, which permits to circumvent the inherent analytical complexity of this model. This is a new and useful result, as it is key for evaluating several important performance metrics of different wireless communication systems and also permits to readily compute the moments of the output SNR. Thus, we obtain simple exact expressions for the energy detection performance in Beckmann fading channels, both in terms of the receiver operating characteristic (ROC) curve and of the area under ROC curve. We also analyze the outage probability in interference limited systems affected by Beckmann fading, as well as the outage probability of secrecy capacity in wiretap Beckmann fading channels. Monte Carlo simulations have been performed to validate the derived expressions.
\end{abstract}

Index Terms-Beckmann fading, Maximal Ratio Combining (MRC), Square-Law Combining (SLC), Energy Detection, Receiver Operating Characteristic (ROC), Secrecy Capacity.

\section{INTRODUCTION}

Because of the presence of multiple scatterers, the radio signal in wireless environments is built from the superposition of a number of individual waves, each with a certain amplitude and phase. Thus, the complex baseband signal (or, equivalently, field) of a wireless channel can be expressed as

$$
v \triangleq R e^{j \Phi}=\sum_{i=1}^{n} A_{i} e^{j \phi_{i}},
$$

where $R$ is the amplitude and $\Phi$ the phase of the resulting signal, and $A_{i}$ and $\phi_{i}$ denotes, respectively, the amplitudes and phases of the individual components. By assuming a sufficiently large number of paths, and by virtue of the Central Limit Theorem [1], the received signal can be modeled as a complex Gaussian random variable (RV), which can thence be

J. P. Peña-Martín and J. M. Romero-Jerez are with the Department of Electronic Technology, F. J. López-Martínez is with the Department of Communications Engineering, E.T.S.I. Telecomunicación, University of Málaga, 29071 Málaga, Spain (e-mail: jppena@uma.es, romero@dte.uma.es, fjlopezm@ic.uma.es).

The material in this paper was submitted, in part, to the IEEE 85th Vehicular Technology Conference. written as $v=X+j Y$. This topic was originally addressed by Beckmann [2,3] in its more general form by assuming arbitrary mean and variance for the real and imaginary parts of $v$, i.e., $X \sim \mathcal{N}\left(\mu_{x}, \sigma_{x}^{2}\right)$ and $Y \sim \mathcal{N}\left(\mu_{y}, \sigma_{y}^{2}\right)$, being $X$ and $Y$ independent ${ }^{1}$. This corresponds to the most accurate way to characterize the scattering of electromagnetic waves from rough surfaces [5], on which the distribution of the received signal envelope $R=|v|$ is that of the modulus of a complex Gaussian RV.

The Beckmann distribution includes the most popular classical fading models used in practice, such as the Rician [6], Hoyt (Nakagami- $q$ ) [7] and Rayleigh distributions, as particular cases. Unlike other state-of-the-art envelope fading models $[8,9]$, the effect of imbalances in the line-of-sight (LOS) and non-LOS (NLOS) components is considered at the same time. Thus, the Beckmann fading model effectively captures the correlation between the amplitudes and phases of each ray component in (1) [10]. Besides, it allows for modeling LOS propagation conditions with a Hoyt-distributed diffuse component, which accurately fit field measurements in different scenarios [10-12]. However, the distribution of the signal envelope $R$ has a very complicated form, being its chief probability functions, namely probability density function (PDF) and cumulative density function (CDF), unavailable in closed-form $[4,5,13]$. This fact has hindered the performance evaluation of wireless communication systems operating under this otherwise intuitive and physically-justified fading model. For this reason, and despite remarkable efforts have been made in order to analyze different performance metrics such as capacity, error probability, level crossing statistics and outage probability under Beckmann fading [14-16], there are still many communication-theoretic open problems which remain unexplored when Beckmann fading is considered.

The contribution of this paper is two-fold: we derive a closed-form expression for the generalized moment generating function (MGF) of the signal-to-noise ratio (SNR) under Beckmann fading, which is given in terms of elementary functions and from which the moments of the output SNR can be readily obtained. We then illustrate the applicability of the generalized MGF in three different scenarios of interest, and for which the performance under Beckmann fading has been

\footnotetext{
${ }^{1}$ As argued in [4], the assumption of independence does not cause any loss of generality. Should $X$ and $Y$ be correlated, there exists a linear transformation (equivalent to rotating the axis a certain angle $\varphi$ ) which yields a pair of uncorrelated Gaussian RVs $X^{\prime}$ and $Y^{\prime}$ with non-zero mean and non-identical variances. The symbol $\sim$ means statistically distributed as.
} 
largely unknown: (1) energy detection, (2) outage probability with co-channel interference in interference limited scenarios, and (3) physical layer security.

The remainder of this paper is structured as follows: in Section II, we derive the generalized MGF of the SNR of the Beckmann fading, which will enable the analysis of the aforementioned scenarios. Then, in Section III we provide analytical expressions for the probability of detection of unknown signals in Beckmann fading channels, as well as for the area under the receiver operating characteristic (ROC) figure of merit. Section IV is devoted to analyze the outage probability in Beckmann fading channels in the presence of interference. Section $\mathrm{V}$ investigates the physical layer security performance, in terms of the outage probability of secrecy capacity, when the wiretap link is affected by Beckmann fading. Numerical results are given in Section VI, whereas the main conclusions are outlined in Section VII.

\section{STATISTICAL ANALYSiS}

\section{A. Preliminary definitions}

We first present some definitions which will be of later use in the analysis.

Definition 1 (Generalized MGF): Let $\xi$ be a continuous non-negative random variable with $\operatorname{PDF} f_{\xi}(\cdot)$. The generalized MGF of $\xi$ is defined as

$$
\phi_{\xi}^{(n)}(s) \triangleq E\left\{\xi^{n} e^{\xi s}\right\}=\int_{0}^{\infty} x^{n} e^{x s} f_{\xi}(x) d x,
$$

where $E\{\cdot\}$ denotes the expectation operator.

In the sequel, we will assume $n \in \mathbb{N}$. Note that in this case the generalized MGF coincides with the $n^{\text {th }}$ order derivative of the $\operatorname{MGF} \phi_{\xi}(s) \triangleq E\left\{e^{\xi s}\right\}=\phi_{\xi}^{(0)}(s)$, and the $n^{\text {th }}$ order moment of $\xi$ is readily obtained as $\mu_{n} \triangleq E\left\{\xi^{n}\right\}=\phi_{\xi}^{(n)}(0)$.

Definition 2 (Beckmann envelope distribution): Let $v=X+j Y$ be a complex Gaussian RV such as $X \sim \mathcal{N}\left(\mu_{x}, \sigma_{x}^{2}\right)$ and $Y \sim \mathcal{N}\left(\mu_{y}, \sigma_{y}^{2}\right)$, being $X$ and $Y$ independent. Then, the $\mathrm{RV} R=|v|$ representing the signal envelope in (1) is said to be Beckmann distributed, and its $\mathrm{PDF}$ is given by [2, eq. (31)]

$$
f_{R}(v)=\frac{v}{2 \pi \sigma_{x} \sigma_{y}} \int_{0}^{2 \pi} e^{-\frac{\left(v \cos (\theta)-\mu_{x}\right)^{2}}{2 \sigma_{x}^{2}}-\frac{\left(v \sin (\theta)-\mu_{y}\right)^{2}}{2 \sigma_{y}^{2}}} d \theta .
$$

The Beckmann distribution includes the most popular fading distributions such as Rayleigh, Hoyt and Rician as particular cases, by simply specializing the four parameters $\mu_{x}, \mu_{y}, \sigma_{x}$ and $\sigma_{y}$. For convenience of discussion and in order to facilitate the physical interpretation, an alternative definition for the parameters of the Beckmann distribution is usually preferred [17, eq. (2.39)].

Definition 3 (Beckmann distribution parameters): Let $R$ be a Beckmann distributed RV with parameters $\mu_{x}, \mu_{y}, \sigma_{x}^{2}$ and $\sigma_{y}^{2}$. Then, the following parameters are defined in order to univoquely define the Beckmann distribution:

$$
\begin{aligned}
& q^{2} \triangleq \frac{\sigma_{x}^{2}}{\sigma_{y}^{2}}, \quad \quad r^{2} \triangleq \frac{\mu_{x}^{2}}{\mu_{y}^{2}}, \quad K \triangleq \frac{\mu_{x}^{2}+\mu_{y}^{2}}{\sigma_{x}^{2}+\sigma_{y}^{2}}, \\
& \Omega \triangleq \mu_{x}^{2}+\mu_{y}^{2}+\sigma_{x}^{2}+\sigma_{y}^{2} .
\end{aligned}
$$

Table I

CONNECTIONS BETWEEN BECKMANN FADING AND OTHER FADING MODELS IN THE LITERATURE. THE BECKMANN FADING PARAMETERS ARE UNDERLINED TO AVOID CONFUSION WITH THE SPECIAL CASES.

\begin{tabular}{c|ll}
\hline Channels & \multicolumn{2}{|l}{ Beckmann Fading Parameters } \\
\hline \hline One-sided Gaussian & $\underline{r}=1, \quad \underline{q}=0, \quad \underline{K}=0$ \\
\hline Rayleigh & $\underline{r}=1, \quad \underline{q}=1, \quad \underline{K}=0$ \\
\hline Hoyt (Nakagami- $q)$ & $\underline{r}=1, \quad \underline{q}=q, \quad \underline{K}=0$ \\
\hline Rician with parameter $K$ & $\underline{r}=1, \quad \underline{q}=1, \quad \underline{K}=K$ \\
\hline LOS with diffuse Hoyt & $\underline{r}=1, \quad \underline{q}=q, \quad \underline{K}=K$ \\
\hline Symmetrical $\eta-\kappa[18]$ & $\underline{r}=\eta, \quad \underline{q}=\eta, \quad \underline{K}=\kappa$ \\
\hline Asymmetrical $\eta-\kappa[19]$ & $\underline{r}=0, \quad \underline{q}=\eta, \quad \underline{K}=\kappa$ \\
\hline \hline
\end{tabular}

The parameter $K$ accounts for the ratio between the LOS and non-LOS (NLOS) power, similarly to the definition of the Rician $K$ parameter. In the same way, the parameter $q$ measures the power imbalance between the in-phase (I) and quadrature (Q) NLOS components as in the Hoyt (Nakagami$q$ ) fading model. The parameter $r$ also indicates a power imbalance between the I and Q components, but now for the LOS component ${ }^{2}$. Finally the parameter $\Omega$ can be regarded as the average received power $\Omega=E\left\{|v|^{2}\right\}=E\left\{R^{2}\right\}$. The connection between the Beckmann distribution and the special cases included therein can easily be set by using the previous definitions for $q, r$ and $K$, and is formally stated in Table I.

\section{B. Beckmann fading statistics}

In most communication-theoretic scenarios the distribution of the SNR is of more convenience than the distribution of the signal envelope, as many different performance metrics are a function of the received power or the SNR. We now derive the generalized MGF of the received SNR under Beckmann fading.

Lemma 1: Let $R$ be a Beckmann distributed RV with $E\left\{R^{2}\right\}=\Omega$. Let $\gamma \triangleq R^{2} E_{s} / N_{0}$ be the received SNR under Beckmann fading, where $E_{s}$ is the symbol energy and $N_{0}$ is the one-sided AWGN power spectral density, and let $\bar{\gamma}=\Omega E_{s} / N_{0}$ denote its average. Then, the generalized MGF of $\gamma, \phi_{\gamma}^{(n)}(s)$, is given by (6).

Proof: See Appendix A.

This expression is new in the literature to the best of our knowledge. Unlike the Beckmann PDF or CDF, the generalized MGF of the SNR in Beckmann fading has a closed-form expression. As we will later see, this has important relevance in practice, enabling the analysis of different performance metrics without any increase in complexity when compared to the simpler cases of Rayleigh, Hoyt or Rician fading. It is easy to check that when $n=0$, (6) reduces to the MGF expression given in [17, eq. (2.41)].

\footnotetext{
${ }^{2}$ Note that the parameters $q$ and $r$ can take values within the whole range $[0, \infty)$, showing a symmetric behavior in the intervals $[0,1]$ and $[1, \infty)$.
} 


$$
\begin{aligned}
\phi_{\gamma}^{(n)}(s) & =\frac{\left(1+q^{2}\right)(1+K)}{\sqrt{\left[\left(1+q^{2}\right)(1+K)-2 q^{2} \bar{\gamma} s\right]\left[\left(1+q^{2}\right)(1+K)-2 \bar{\gamma} s\right]}} \\
& \times \exp \left[\frac{K \frac{1}{1+r^{2}}\left(1+q^{2}\right) \bar{\gamma} s}{\left(1+q^{2}\right)(1+K)-2 \bar{\gamma} s}+\frac{K \frac{r^{2}}{1+r^{2}}\left(1+q^{2}\right) \bar{\gamma} s}{\left(1+q^{2}\right)(1+K)-2 q^{2} \bar{\gamma} s}\right] \\
& \times \bar{\gamma}^{n} \frac{n !}{2^{n}} \sum_{k=0}^{n} \frac{(2 k) !(2(n-k)) !}{k !(n-k) !}\left(\frac{q^{2}}{\left(1+q^{2}\right)(1+K)-2 q^{2} \bar{\gamma} s}\right)^{k}\left(\frac{1}{\left(1+q^{2}\right)(1+K)-2 \bar{\gamma} s}\right)^{n-k} \\
& \times\left[\sum_{m=0}^{k} \frac{1}{(k-m) !(2 m) !}\left(\frac{\left.\left.\frac{2 r^{2}}{1+r^{2}} \frac{\left(1+q^{2}\right)^{2}}{\left(1+q^{2}\right)(1+K)-2 q^{2} \bar{\gamma} s}\right)^{m}\right]}{\left(1+\frac{2}{\left(1+r^{2}\right)}\left(1+q^{2}\right)^{2} K(1+K)\right.}\right)^{m}\right] . \\
& \times\left[\sum_{m=0}^{n-k} \frac{1}{\left(1+q^{2}\right)(1+K)-2 \bar{\gamma} s}\right)^{m-k-m) !(2 m) !}
\end{aligned}
$$

Corollary 1: Let $\gamma$ be the received SNR under Beckmann fading. Then, the $n^{\text {th }}$ order moment of $\gamma$ is given by

$$
\begin{aligned}
\mu_{n} & =\bar{\gamma}^{n} \frac{n !}{2^{n}\left(1+q^{2}\right)^{n}(1+K)^{n}} \sum_{k=0}^{n} \frac{(2 k) !(2(n-k)) !}{k !(n-k) !} q^{2 k} \\
& \times\left[\sum_{m=0}^{k} \frac{1}{(k-m) !(2 m) !}\left(\frac{2 r^{2}}{1+r^{2}} \frac{\left(1+q^{2}\right)}{q^{2}} K\right)^{m}\right] \\
& \times\left[\sum_{m=0}^{n-k} \frac{1}{(n-k-m) !(2 m) !}\left(\frac{2}{\left(1+r^{2}\right)}\left(1+q^{2}\right) K\right)^{m}\right] .
\end{aligned}
$$

Proof: The proof follows directly from (6) by just considering the equality $\mu_{n}=\phi_{\gamma}^{(n)}(0)$.

To the authors' knowledge, this result has not been reported previously in the literature. The moments of the output SNR permits to evaluate high-order metrics of the SNR, which can be easily derived in closed-form from our result. This is particularly relevant in situations where the PDF is not known in closed-form (i.e., the case of Beckmann fading), as much statistical information can be extracted from them. These metrics include the skewness and the kurtosis, as well as the amount of fading (AoF). The AoF is defined as the SNR variance normalized to its squared mean, and represents a measure of the severity of a fading channel. This parameter provides information about the system performance in a simple way as, typically, the higher its value, the worse the system performance. Using (7), the AoF can be found to be given in Beckmann fading by

$$
\mathrm{AoF}=\frac{2\left(1+r^{2}\right)\left(1+q^{4}\right)+4\left(1+r^{2} q^{2}\right)\left(1+q^{2}\right) K}{\left(1+r^{2}\right)\left(1+q^{2}\right)^{2}(1+K)^{2}} .
$$

It can be checked that for $K=0$ (Hoyt fading), (8) reduces to the AoF expression given in [17, eq. (2.14)], while for $q=1$ (Rician fading), (8) reduces to [17, eq. (2.19)]. Note that,from the obtained expression, it is clear that as $K$ increases, the AoF is reduced, as expected. Also, it is interesting to note that, when $q=1$, parameter $r$ disappears from the expression, i.e., the in-phase and quadrature imbalance of the LOS component has no effect in this case.

\section{Performance Evaluation for energy detection}

The problem of detecting a signal of unknown form in the presence of noise is a classical problem in communication theory, ever since the detection of unknown but deterministic signals using an energy-measuring device was studied by Urkowitz [20]. In this work, we build upon the approaches introduced in $[21,22]$ to analyze the performance of energy detectors under Beckmann fading, providing analytical expressions for the most useful performance metrics: the probability of detection and the area under the ROC curve (AUC). For the reader's convenience, we present the underlying statistical problem before presenting our main results for energy detection under Beckmann fading.

\section{A. Hypothesis Test}

In order to detect an unknown deterministic signal $s(t)$, two hypotheses are considered for the receive signal $y(t)$ [20],

$$
\begin{array}{ll}
H_{0}: & y(t)=n(t), \\
H_{1}: & y(t)=h s(t)+n(t),
\end{array}
$$

where $h$ denotes the channel gain and $n(t)$ is the onesided AWGN with power spectral density $N_{0}$. Under the null hypothesis $H_{0}$, the channel is not occupied by any user signal and there is only noise. The alternative hypothesis $H_{1}$ denotes signal presence and both noise and the transmitted signal are detected at the receiver. Initially, $h \cdot s(t)$ is considered to be approximately constant during the evaluation time interval. Hence, after pre-filtering and sampling, the energy detection decision variable [20,21], $Y=\left(2 / N_{0}\right) \int_{0}^{T} y^{2}(t) d t$, follows a central $\chi_{2 u}^{2}$ distribution under $H_{0}$, and a noncentral $\chi_{2 u}^{2}(2 \gamma)$ distribution under $H_{1}$, where $2 \gamma$ is the noncentrality parameter, $\gamma$ denotes the instantaneous SNR and $u=T W$ is the product of the one-sided bandwidth $W$ and the observation time interval $T$, which can be easily adjusted so that $u \in \mathbb{N}$. The probability of detection is defined as $P_{d}=\operatorname{Pr}\left\{Y>\lambda \mid H_{1}\right\}$, whereas the probability of false alarm is defined as $P_{f}=\operatorname{Pr}\left\{Y>\lambda \mid H_{0}\right\}$, and can be evaluated as [23]

$$
P_{d}=Q_{u}(\sqrt{2 \gamma}, \sqrt{\lambda})
$$




$$
P_{f}=\frac{\Gamma(u, \lambda / 2)}{\Gamma(u)}=e^{-\lambda / 2} \sum_{k=0}^{u-1} \frac{(\lambda / 2)^{k}}{k !},
$$

where $\lambda$ is the energy detection threshold, $Q_{u}(.,$.$) is the u^{t h}$ order generalized Marcum- $Q$ function [17, eq. (4.60)], $\Gamma(a, x)$ is the upper incomplete Gamma function [24, eq. (8.350.2)] and $\Gamma(a)=\Gamma(a, 0)$ is the Gamma function. The last equality in (11) is obtained when $u$ is a positive integer, considering [24, eq. (8.352.2)]. Note that (10) actually denotes the probability of detection conditioned to a particular realization $h$ of the fading channel, or, equivalently, a particular realization of the instantaneous SNR $\gamma$.

\section{B. Average detection probability in Beckmann fading}

The average probability of detection $\overline{P_{d}}$ can be calculated by averaging the instantaneous probability of detection given in (10) over the SNR realizations. Thus, assuming that the PDF of the SNR is denoted as $f_{\gamma}(\gamma)$, we can write

$$
\overline{P_{d}}=\int_{0}^{\infty} Q_{u}(\sqrt{2 \gamma}, \sqrt{\lambda}) f_{\gamma}(\gamma) d \gamma .
$$

In the following derivations, we leverage the approach in [21] to obtain an analytical expression for (12), on which using the series expansion of the generalized Marcum- $Q$ function given by

$$
Q_{u}(\sqrt{2 \gamma}, \sqrt{\lambda})=e^{-\gamma} \sum_{n=0}^{\infty} \frac{\gamma^{n}}{n !} \frac{\Gamma(u+n, \lambda / 2)}{\Gamma(u+n)},
$$

the average detection probability can be obtained by combining (13) and (12), yielding

$$
\begin{aligned}
\overline{P_{d}} & =\left.\sum_{n=0}^{\infty} \frac{\Gamma(u+n, \lambda / 2)}{n ! \Gamma(u+n)} \phi_{\gamma}^{(n)}(s)\right|_{s=-1} \\
& =\left.\sum_{n=0}^{\infty} \sum_{q=0}^{u+n-1}\left(\frac{\lambda}{2}\right)^{q} \frac{e^{-\lambda / 2}}{n ! q !} \phi_{\gamma}^{(n)}(s)\right|_{s=-1},
\end{aligned}
$$

where $\phi_{\gamma}^{(n)}(s)$ is the generalized MGF of $\gamma$. Then, the average detection probability of an energy detector in Beckmann fading can be obtained by plugging (6) into (14). The so-called ROC curve is obtained by representing $\overline{P_{d}}$ vs. $P_{f}$, for different values of $u$ and $\lambda$. Note that $\overline{P_{f}}=P_{f}$, as given in (11), as the false alarm probability does not depend on the SNR. Alternatively, a complementary ROC curve is obtained by representing $\overline{P_{m}}=1-\overline{P_{d}}$, defined as the probability of missed detection (i.e., failing to detect a signal which is present in the channel) vs. $P_{f}$, which can be interpreted in a simple way: the lower the complementary ROC curve, the better system performance for energy detection. The convergence of the infinite series expression evaluated when computing $\overline{P_{m}}$ (equivalently $\overline{P_{d}}$ ) is analyzed in Appendix B.

\section{Average detection probability with diversity combining}

In the previous analysis, we assumed a single-antenna configuration for the energy detection receiver. However, in order to improve the SNR in the presence of fading, diversity combining techniques which make use of multiple antennas are widely used. Depending on the strategy used for the combination of the multiple signals at each branch, different performances can be attained. We now study the effect of using maximal ratio combining (MRC) and square-law combining (SLC) on the energy detection performance, considering receivers equipped with $L$ antennas.

1) Maximal Ratio Combining (MRC): Because it is a coherent combining method, this technique requires channel knowledge, and the received signals are combined before the detection process (pre-detection combining). Thus, an important advantage of the energy detection (i.e., the unnecessary channel knowledge) is lost at the expense of an expectable better performance. However, the study of the energy detection performance under MRC is indeed useful as an upper-bound indicator of the achievable performance [25].

In MRC, the instantaneous combined SNR is given by $\gamma_{M R C}=\sum_{k=1}^{L} \gamma_{k}$, where $\gamma_{k}$ is the instantaneous SNR at the $k^{t h}$ branch. Assuming that the receive signals at every branch are independent, the MGF of $\gamma_{M R C}$ is $\phi_{M R C}(s)=$ $\prod_{k=1}^{L} \phi_{\gamma_{k}}(s)$. Then, with the help of the multinomial theorem [26, eq. 24.1.2] we can write

$$
\phi_{M R C}^{(n)}(s)=\sum_{\tau(n, L)} \frac{n !}{q_{1} ! q_{2} ! \cdots q_{L} !} \phi_{\gamma_{1}}^{\left(q_{1}\right)}(s) \phi_{\gamma_{2}}^{\left(q_{2}\right)}(s) \cdots \phi_{\gamma_{L}}^{\left(q_{L}\right)}(s),
$$

where $\tau(n, L)$ is defined as the set of $L$-tuples such that $\tau(k, L)=\left\{\left(q_{1}, q_{2}, \cdots, q_{L}\right): q_{m} \in \mathbb{N}, \sum_{m=1}^{L} q_{m}=k\right\}$. Combining now (14) and (15), $\overline{P_{d}}$ becomes

$$
\begin{aligned}
& \overline{P_{d, M R C}}=\left.\sum_{n=0}^{\infty} \frac{\Gamma(u+n, \lambda / 2)}{n ! \Gamma(u+n)} \phi_{M R C}^{(n)}(s)\right|_{s=-1}= \\
& \left.\sum_{n=0}^{\infty} \sum_{\tau(n, L)} \sum_{m=0}^{u+n-1} \frac{\left(\frac{\lambda}{2}\right)^{m} e^{-\lambda / 2}}{m !} \frac{\phi_{\gamma_{1}}^{\left(q_{1}\right)}(s)}{q_{1} !} \cdots \frac{\phi_{\gamma_{L}}^{\left(q_{L}\right)}(s)}{q_{L} !}\right|_{s=-1},
\end{aligned}
$$

where in the last equality we assumed that $u \in \mathbb{N}$. Under this scheme, $\overline{P_{f}}$ is also given by

$$
\overline{P_{f, M R C}}=\frac{\Gamma(u, \lambda / 2)}{\Gamma(u)} .
$$

2) Squared Law Combining (SLC): This is a post-detection combining method, that is, the decision variable is combined after sampling [1, sec. 5.4]. Again, $\gamma_{S L C}=\sum_{k=1}^{L} \gamma_{k}$ as in the MRC case, therefore $\phi_{S L C}^{(n)}=\phi_{M R C}^{(n)}$ as given in (15). However, the number of samples to be considered is not $u$ but $L u$. Hence, $\overline{P_{d}}$ and $\overline{P_{f}}$ become, respectively [21],

$$
\begin{gathered}
\overline{P_{d, S L C}}=\left.\sum_{n=0}^{\infty} \frac{\Gamma(L u+n, \lambda / 2)}{n ! \Gamma(L u+n)} \phi_{S L C}^{(n)}(s)\right|_{s=-1}, \\
\overline{P_{f, S L C}}=\frac{\Gamma(L u, \lambda / 2)}{\Gamma(L u)} .
\end{gathered}
$$




\section{Area under the ROC curve}

An alternative method to evaluate and compare the system performance for energy detection is the area under the ROC curve (AUC), as introduced in [25]. The use of an alternative figure of merit to describe the performance of an energy detector is motivated by the difficulty of comparing the ROC curves for two different detectors, as their ROC curves may cross. Besides, while ROC curves plot the detection probability vs. the false alarm probability, the AUC encapsulates all this information in a single performance metric.

The AUC (or equivalently, the complementary AUC if $P_{m}$ is used) is defined as

$$
A(\gamma) \triangleq \int_{0}^{1} P_{d}(\gamma, \lambda) d P_{f}(\lambda) .
$$

Note that the threshold value $\lambda$ varying from 0 to $\infty$ implies that $P_{d}$ varies from 1 to 0 . The AUC metric, however, takes values in the range $[0.5,1]$. When conditioned to a particular channel state (i.e. conditioned to a given $\gamma$ ), the AUC is expressed as [22, eq. (10)]

$$
A(\gamma)=1-\sum_{q=0}^{u-1} \sum_{n=0}^{q}\left(\begin{array}{c}
q+u-1 \\
q-n
\end{array}\right)\left(\frac{1}{2}\right)^{n+q+u} \frac{1}{n !} \gamma^{n} e^{-\gamma / 2}
$$

Therefore, the average AUC for $u \in \mathbb{N}$ can be obtained by averaging (21) over all possible channel states, yielding

$$
\begin{aligned}
\bar{A} & =1-\sum_{q=0}^{u-1} \sum_{n=0}^{q}\left(\begin{array}{c}
q+u-1 \\
q-n
\end{array}\right)\left(\frac{1}{2}\right)^{n+q+u} \frac{1}{n !} \\
& \times \int_{0}^{\infty} \gamma^{n} e^{-\gamma / 2} f_{\gamma}(\gamma) d \gamma \\
& =1-\left.\sum_{q=0}^{u-1} \sum_{n=0}^{q}\left(\begin{array}{c}
q+u-1 \\
q-n
\end{array}\right)\left(\frac{1}{2}\right)^{n+q+u} \frac{1}{n !} \phi_{\gamma}^{(n)}(s)\right|_{s=-\frac{1}{2}} .
\end{aligned}
$$

Note that (22), which was originally given in $[22,13]$, allows for obtaining the average AUC for any arbitrary fading distribution in a simple way, provided that the generalized MGF is known. Thus, we obtain the average AUC when considering Beckmann fading by using (6) in (22), which is a new result that has not been previously reported, to the authors' knowledge. This result can be extended to include multiple receive antennas performing a certain diversity scheme in the same way as in the detection probability result presented in the previous subsection. We must note that in order to evaluate the average AUC, only a finite number of elementary functions is needed; thus, the expression for the average AUC here obtained is of similar complexity to the ones obtained for the Rician scenario [22], and simpler than the ones obtained in [27] for Hoyt fading, which are particular cases of our result. When compared to the available results for generalized fading channels [28-30] such as $\kappa-\mu, \eta-\mu$ and $\alpha-\mu$, they are all given in terms of a finite summation. However, the evaluation of hypergeometric or Meijer- $G$ functions is not required in our case unlike when considering $\eta-\mu$ or $\alpha-\mu$ fading and $u \in \mathbb{N}$.

\section{OUTAGE PROBABILITY IN INTERFERENCES LIMITED SCENARIOS}

We now consider the problem of evaluating the outage probability (OP) in an interference limited scenario, considering a single-antenna transmitter and a $L$-antenna receiver, as well as $N$ interfering signals. In this scenario, the receive baseband vector before combining is given by [31]

$$
\mathbf{r}=\mathbf{h}_{\mathbf{0}} b_{0}+\sum_{i=1}^{N} \sqrt{P_{i}} \mathbf{h}_{\mathbf{i}} b_{i}
$$

where all the vectors have dimension $L, \mathbf{h}_{\mathbf{0}}$ is the channel gain of the desired signal, $\mathbf{h}_{\mathbf{i}}$ is the channel gain of the $i^{t h}$ interfering signal, $b_{0}$ and $b_{i}$ are, respectively, the transmitted symbols from the desired and $i^{\text {th }}$ interfering user which, for simplicity, we suppose to be normalized, $\left|b_{j}\right|=1, j=0,1, \ldots, N$. $P_{i}$ is the received power for the $i^{t h}$ interfering signal. We also assume that all signals undergo slow flat fading, and perfect knowledge of the instantaneous channel state for the desired signal is considered, thus allowing the use of a coherent detection technique such as MRC.

In order to consider the most general situation for the fading affecting the desired signal, we assume that the $L$ components of $\mathbf{h}_{\mathbf{0}}$ are independent and non-identically distributed (i.n.d.) complex Gaussian random variables where both the in-phase and quadrature components have arbitrary mean and variance, i.e., $\left|h_{0 j}\right|$, being $h_{0 j}$ the $j^{\text {th }}$ component of $\mathbf{h}_{\mathbf{0}}$, is Beckmann distributed. We also assume that the set of $N$ interfering signals undergoes Rayleigh fading, which is coherent with the assumption that co-channel interference arrives at the receiver through a NLOS path. Thus, the components of $\mathbf{h}_{\mathbf{i}}$ are i.i.d. complex Gaussian random variables with zero mean and unit variance.

After MRC combining, the signal-to-interference ratio becomes [32, eq. (5)]

$$
\Gamma=\frac{X}{Y}
$$

with

$$
\begin{gathered}
X=\sum_{j=1}^{L}\left|h_{0 j}\right|^{2}, \\
Y=\sum_{i=1}^{N} P_{i}\left|\frac{\mathbf{h}_{0}^{H} \mathbf{h}_{i}}{\left\|\mathbf{h}_{0}\right\|}\right|^{2} .
\end{gathered}
$$

The superscript ${ }^{H}$ represents the Hermitian operator for a complex vector. It is demonstrated in [33] that if the elements of $\mathbf{h}_{\mathbf{i}}$ are i.i.d. zero mean unit variance complex Gaussian random variables, then $\nu_{i}=\mathbf{h}_{0}^{H} \mathbf{h}_{i} /\left\|\mathbf{h}_{0}\right\|$ and $\mathbf{h}_{\mathbf{0}}$ are mutually independent and $\nu_{i}$ is a zero mean unit variance complex Gaussian random variable. Hence, $Y$ is a sum of exponential random variables.

In an interference-limited system, given a threshold $\beta$, the outage probability can be calculated as

$$
P_{\text {out }}=\operatorname{Pr}\{\Gamma<\beta\}=1-\int_{0}^{\infty} F_{Y}(x \mid \beta) f_{X}(x) d x,
$$


where $F_{Y}(\cdot)$ is the CDF of the output interference and $f_{X}(\cdot)$ is the PDF of the output signal from the desired user. As it is demonstrated in [34, eq. (14)], $P_{\text {out }}$ can be calculated as

$$
\begin{aligned}
P_{\text {out }} & =\sum_{i=1}^{J} \sum_{j=1}^{n_{i}} A_{i j} \sum_{k=0}^{n_{i}-j} \frac{1}{\left(\beta P_{i}\right)^{k}} \\
& \times\left.\sum_{\tau(k, L)} \prod_{m=1}^{L} \frac{1}{q_{m} !} \phi_{\gamma_{m}}^{\left(q_{m}\right)}(s)\right|_{s=-1 /\left(\beta P_{i}\right)},
\end{aligned}
$$

where $\gamma_{m}$ represents in this case the received power of the desired signal at antenna $m$, undergoing Beckmann fading, $J$ is the number of different interfering signals, each one with multiplicity $n_{i}$, therefore $\sum_{i=1}^{J} n_{i}=N, \tau(k, L)$ was defined in (15) and

$$
\begin{aligned}
A_{i j} & =(-1)^{j-1} \sum_{\Omega_{A}} \prod_{k=1, k \neq i}^{J}\left(\begin{array}{c}
n_{k}+q_{k}-1 \\
n_{k}-1
\end{array}\right) \\
& \times \frac{P_{k}^{q_{k}} P_{i}^{n_{k}}}{\left(P_{i}-P_{k}\right)^{n_{k}+q_{k}}},
\end{aligned}
$$

where now $\Omega_{A}$ is the set of $J$-tuples such that $\Omega_{A}=\left\{\left(q_{1}, \cdots, q_{J}\right): q_{k} \in \mathbb{N}, q_{i}=0, \sum_{k=1}^{J} q_{k}=j-1\right\}$,

and $\phi_{\gamma_{m}}^{(n)}(\cdot)$ represents the generalized Beckmann MGF. Therefore, as a direct application of Lemma 1 we obtain the desired outage probability in closed-form by simply introducing (6) into (28). This is also a new result in the literature to the best of our knowledge.

\section{PHYSICAL LAYER SECURITY}

In this section, we aim at analyzing the secure communication between two legitimate peers (Alice and Bob, being Alice the transmitter) over a wireless channel in the presence of an external eavesdropper (Eve) that observes their communication through a different link. Specifically, we use $\gamma_{b}$ to denote the instantaneous SNR at Bob, and $\gamma_{e}$ to denote the instantaneous SNR received at Eve. In this set-up, the secrecy capacity $C_{s}$ for a Gaussian wiretap channel is defined as [35]

$$
\begin{aligned}
C_{s} & =\max \left\{C_{b}-C_{e}, 0\right\}, \\
& =\max \left\{\log _{2}\left(1+\gamma_{b}\right)-\log _{2}\left(1+\gamma_{e}\right), 0\right\},
\end{aligned}
$$

which can be regarded as the maximum rate that can be transmitted through the legitimate link while maximizing Eve's uncertainty about the transmitted message. For simplicity, we assumed a normalized bandwidth $B=1$ in the previous capacity definitions.

As in [36], we assume perfect channel state information (CSI) at Alice and Bob for the desired (legitimate) channel, but no CSI knowledge about the wiretap channel. However, Eve indeed has access to perfect CSI for the wiretap channel. In this situation, it is not possible to guarantee perfect secrecy because of the absence of Eve's CSI at Alice; instead, Alice transmits at a rate $R_{s}$ and assumes a certain capacity for the wiretap link $\hat{C}_{e}=C_{b}-R_{s}$, so we have a probabilistic measure of the link security given by

$$
\mathcal{P}_{s} \triangleq \operatorname{Pr}\left\{C_{s} \geq R_{s}\right\}=1-\operatorname{Pr}\left\{C_{s}<R_{s}\right\} .
$$

This metric can be regarded as the probability of a successful secure transmission, whereas the probability $\operatorname{Pr}\left\{C_{s}<R_{s}\right\}$ is usually referred to in the literature as the outage probability of secrecy capacity (OPSC). Then, $\mathcal{P}_{s}$ can be computed as

$$
\begin{aligned}
\mathcal{P}_{s} & =\operatorname{Pr}\left\{\log _{2}\left(\frac{1+\gamma_{b}}{1+\gamma_{e}}\right)>R_{s}\right\} \\
& =\int_{0}^{\infty} f_{\gamma_{e}}(x)\left(\int_{2^{R_{s}}(1+x)-1}^{\infty} f_{\gamma_{b}}(y) d y\right) d x \\
& =\int_{0}^{\infty} f_{\gamma_{e}}(x) \bar{F}_{\gamma_{b}}\left(2^{R_{s}}(1+x)-1\right) d x,
\end{aligned}
$$

where $f_{\gamma_{b}}(\cdot)$ and $f_{\gamma_{e}}(\cdot)$ are the PDFs of $\gamma_{b}$ and $\gamma_{e}$ respectively, whereas $\bar{F}_{\gamma_{b}}(\cdot)$ is the complementary CDF (CCDF) of $\gamma_{b}$. We consider that the legitimate link is affected by Nakagami- $m$ fading. Then, for $m \in \mathbb{N}$ its CCDF is given by:

$$
\bar{F}_{\gamma_{b}}(x)=e^{-x} \sum_{k=0}^{m-1} \frac{x^{k}}{k !} .
$$

Thus, from (32) and (33), after some manipulations, we obtain

$$
\begin{aligned}
\mathcal{P}_{s} & =e^{m \frac{1-2^{R_{s}}}{\bar{\gamma}_{b}}} \sum_{k=0}^{m-1}\left(\frac{m}{\bar{\gamma}_{b}}\right)^{k} \frac{1}{k !} \sum_{n=0}^{k}\left(\begin{array}{l}
k \\
n
\end{array}\right) 2^{R_{s} n}\left(2^{R_{s}}-1\right)^{k-n} \\
& \times\left.\phi_{\gamma_{e}}^{(n)}(s)\right|_{s=-m 2^{R_{s}} / \bar{\gamma}_{b}}
\end{aligned}
$$

where $\phi_{\gamma_{e}}^{(n)}(s)$ is the generalized MGF of the SNR at Eve. Thus, using Lemma 1 we directly have the expression for $\mathcal{P}_{s}$ when the wiretap channel is affected by Beckmann fading. Note that the probability of strictly positive secrecy capacity, defined as $\operatorname{Pr}\left\{C_{s}>0\right\}$, can be computed from (34) by setting $R_{s}=0$.

\section{NUMERICAL RESULTS}

In this section, we present some numerical results of the derived theoretical expressions in order to assess the performance of the considered wireless communication systems experiencing Beckmann fading. We have performed Monte Carlo simulations in order to validate the theoretical results, which are included in the figures with marker dots.

\section{A. Energy detection}

In Fig. 1, complementary ROC curves are depicted in order to analyze the influence of the number of diversity branches in the energy detection performance, as well as the influence of the sampling policy. The ROC curves decrease in a very appreciable manner when the number of branches increases, considering that the received signals per branch experience i.i.d. Beckmann fading. For the same receive array size, the MRC strategy shows a better behavior than SLC, at the expense of requiring a precise channel knowledge. Moreover, the higher the number of diversity branches, the more significant the improvement of MRC. Monte Carlo simulations show a perfect agreement with the theoretical results.

In Fig. 2, the influence of fading severity is evaluated. As expected, the probability of missed detection is much lower in 


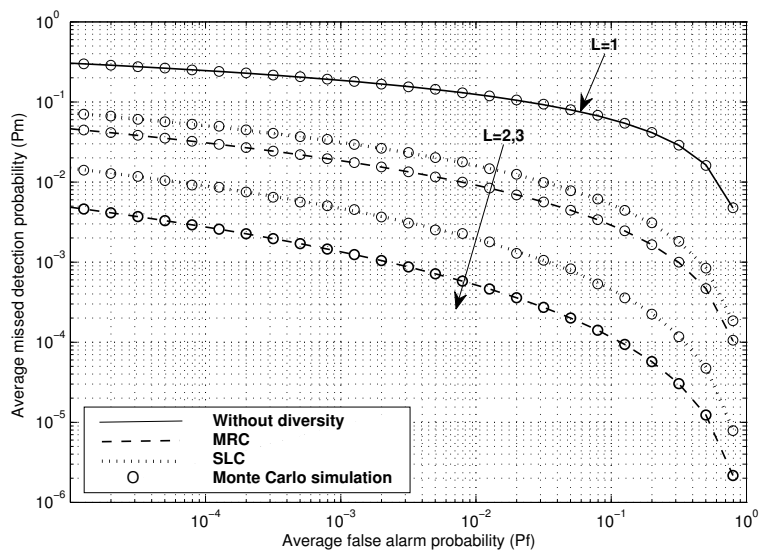

Figure 1. Complementary ROC curve (average $P_{m}$ vs. average $P_{f}$ ) under Beckmann fading for different numbers of receive diversity branches $L$ and sampling policies. The rest of parameters are: average SNR per branch $\bar{\gamma}_{k}=$ $15 \mathrm{~dB} ; q=0.5 ; K=2 ; r=1 ; u=5$.

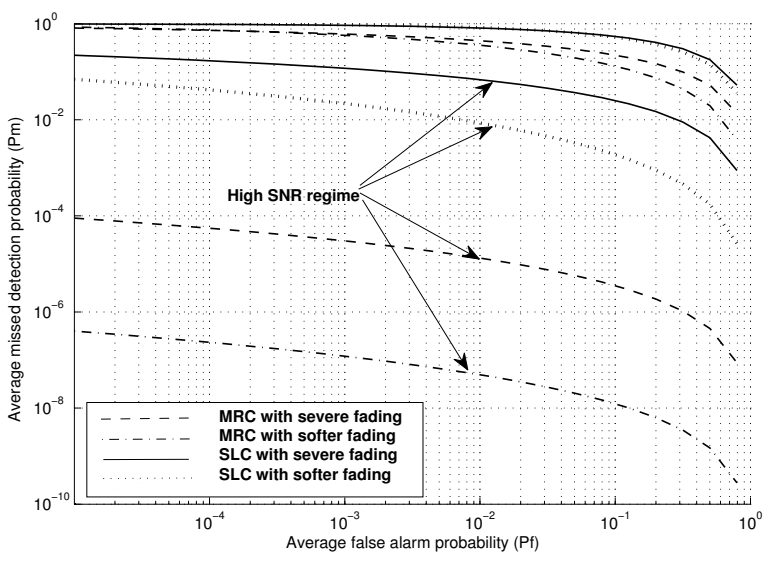

Figure 2. Complementary ROC curve (average $P_{m}$ vs. average $P_{f}$ ) under Beckmann fading for different values of fading severity parameters and average SNRs. 'Severe fading': $\{q=0.1 ; K=0.1\}$; 'Softer fading': $\{q=0.9 ; K=3\}$. Analogously, 'High SNR': $\bar{\gamma}_{k}=25 \mathrm{~dB}$; 'Low SNR': $\bar{\gamma}_{k}=5 \mathrm{~dB}$. The rest of parameters are $L=3 ; r=1 ; u=5$.

the high-SNR regime $\left(\bar{\gamma}_{k}=25 \mathrm{~dB}\right)$; however, it is also relevant how the fading severity and the sampling policy increment their influence at this regime. Conversely, in the low SNRregime $\left(\bar{\gamma}_{k}=5 \mathrm{~dB}\right)$ the differences are smaller. Indeed, MRC shows again a better performance than the SLC policy

In Fig. 3, the performance of the energy detection schemes is now evaluated for the average AUC figure of merit. The MRC and SLC sampling policies have been considered, as well as different parameter values for $K, q$ and $u$. Once again, we can see that the AUC curve for MRC is always above the equivalent one for SLC, which implies a better detection performance. We also observe that increasing the fading severity decreases the detection performance for both MRC and SLC. Finally, as reported in [25], increasing the number of samples $u$ decreases the AUC for a given SNR per branch. The average AUC metric encapsulates the joint effect of $P_{d}$ and $P_{f}$, which both increase with $u$. This behavior is explained by noting that $P_{f}$ increases faster than the detection

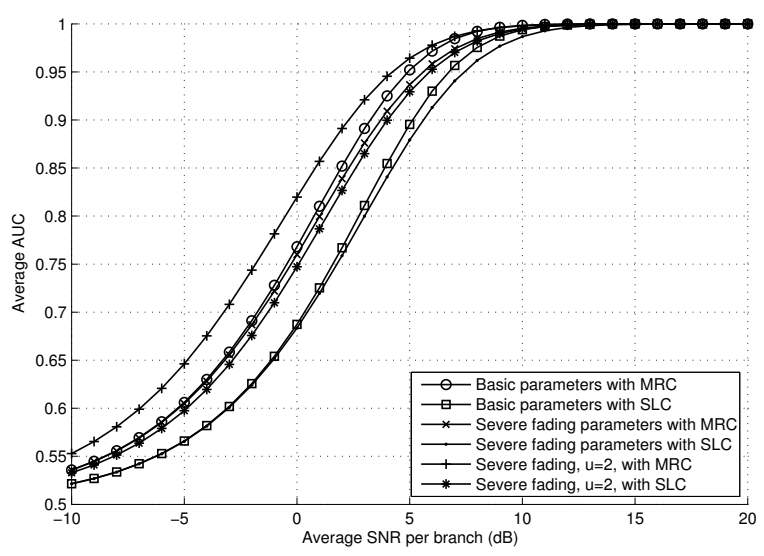

Figure 3. Average AUC vs. average SNR per branch under Beckmann fading, for different values of $K, q$ and $u$ and different sampling policies. 'Basic parameters': $\{q=0.75 ; K=3\}$. 'Severe fading': $\{q=0.25 ; K=1\}$. The value $u=5$ is considered unless otherwise stated. The rest of parameters are $L=3 ; r=1$.

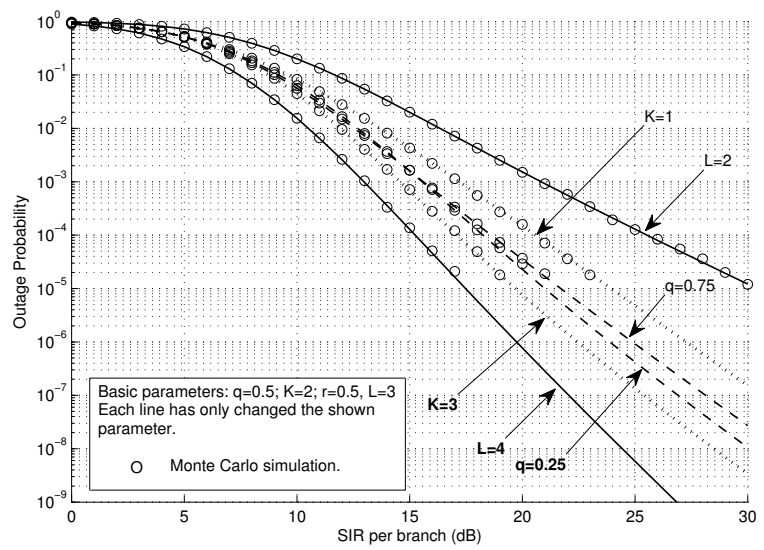

Figure 4. Outage probability vs. average power per branch of the desired signal using MRC combining, for different values of $K, q$ and $L$. Initial parameter values (basic parameters): $q=0.5, K=2, r=0.5, L=3$

probability $P_{d}$, resulting in a lower overall detection capability.

\section{B. Outage probability in interference-limited scenarios}

Results for this scenario are shown in Fig. 4. We consider four interfering signals subject to Rayleigh fading with $J=2$, $n_{1}=2, n_{2}=2$, and average powers per branch, $P_{1}=0.2$, $P_{2}=0.3$ according to the definitions in Section IV. The desired signals at every receive antenna undergo Beckmann fading and are assumed to be i.i.d. Starting with an initial set of parameters labeled as 'basic parameters' ( $q=0.5, K=2$, $r=0.5, L=3$ ), we show results when one of these parameters is varied at a time. It can be observed that increasing the LOS power $(K)$, the number of diversity branches $(L)$ and the circular symmetry of the diffuse component $(q \rightarrow 1)$, improves the OP performance. For the sake of clarity in the figure, we have not included variations on $r$; however, the effect of increasing/decreasing $r$ is similar as increasing/decreasing $q$ (i.e., performance improves as $r \rightarrow 1$ ). It can also be observed 
that Monte Carlo simulations show an excellent agreement with the theoretical data.

\section{Physical layer security}

We now evaluate the physical layer security performance attending to the probability of achieving a secure transmission $\mathcal{P}_{s}$ (non-outage probability), when the wiretap channel is affected by Beckmann fading. As indicated in Section V, we assume that the fading in the legitimate link can be modeled with the Nakagami- $m$ distribution. In Fig. 5 and $6, \mathcal{P}_{s}$ is represented as a function of the average SNR at the legitimate receiver for different values of the fading parameters in both the legitimate and wiretap channel. We consider that the average SNR of the eavesdropper is maintained constant. Fig. 5 represents the non-outage probability for a given threshold $R_{s}=1$, while Fig. 6 show results for the strictly positive secrecy capacity, i.e., $R_{s}=0$. In these figures, we call 'standard fading' to the following set of Beckmann channel parameters: $q=0.9, K=5, r=1, \bar{\gamma}_{e}=0 \mathrm{~dB}$, where $\bar{\gamma}_{e}$ is the average SNR for the eavesdropper. Analogously, we call 'severe fading' to the following set of Beckmann channel parameters: $q=0.1, K=1, r=1, \bar{\gamma}_{e}=0 \mathrm{~dB}$. Both figures show a somewhat different behavior. In the low SNR regime (say $\gamma_{b}<-10 \mathrm{~dB}$ ), Fig, 6 shows that, when the fading experienced by Eve becomes less severe, $\mathcal{P}_{s}$ is also lower for the same average SNR at Bob. The behavior is due to the fact that when $\gamma_{b}$ becomes very low, the eavesdropper conditions are dominant, as we could expect. However, in the high SNR regime the behavior is just the opposite, and the fading experienced by Bob determines the value of $\mathcal{P}_{s}$. In fact, as pointed out in [37], in the high SNR regime the probability of achieving a secure transmission is independent of the distribution of the SNR at Eve, being only affected by its average value $\bar{\gamma}_{e}$. which can shift the curves to the right or to the left. From Fig. 5, we can observe a similar behavior at high SNR regime, obviously shifted by the threshold $R_{s}$ effect. In the lower SNR regime, when $\gamma_{b}$ is no longer dominant, as $\bar{\gamma}_{e}$ is constant and $\mathcal{P}_{s}=\operatorname{Pr}\left\{C_{b}>R_{s}+C_{e}\right\}$, the weight of $C_{e}$ can be limited by $R_{s}$, which does not happen when $R_{s}=0$.

\section{CONCLUSIONS}

In this work, we have found a closed-form expression for the generalized MGF of the SNR in Beckmann channels in terms of elementary functions, from which all the moments have also been derived. In order to demonstrate the usefulness of our result, we have used it to analyze the energy detection performance in Beckmann fading channels, both in terms of the receiver operating characteristic (ROC) and of the area under ROC. We also analyzed the outage probability in interference limited systems affected by Beckmann fading, as well as the probability of secrecy capacity in wiretap Beckmann fading channels.

For the energy detection, numerical results show the influence of the receiver combining strategy and the Beckmann fading parameters. As a general conclusion, any imbalance between the underlying Gaussian RVs inherent to Beckmann fading, either in the LOS (parameter $r$ ) or diffuse (parameter $q$ )

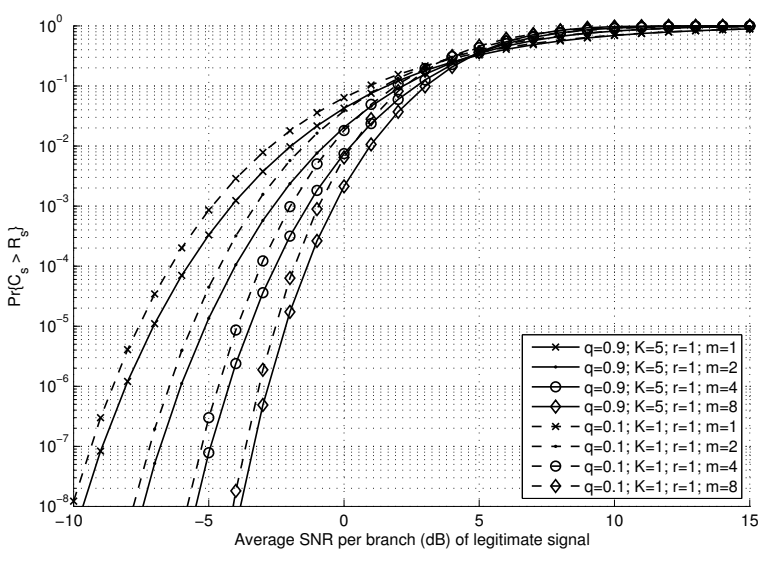

Figure 5. $\mathcal{P}_{s}$ vs. average SNR, for different values of $q, K, r$ (eavesdropper channel) and $m$ (legitimate channel). Parameter values are $\bar{\gamma}_{e}=0 \mathrm{~dB}$ and $R_{s}=1$.

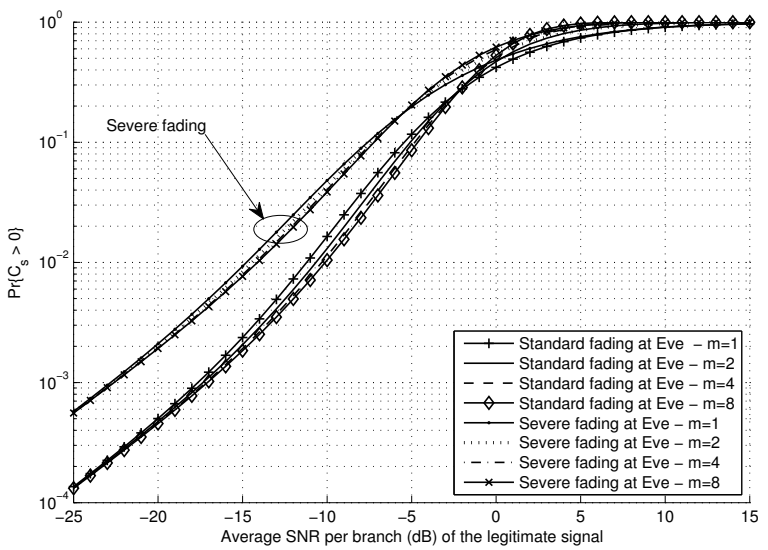

Figure 6. $\mathcal{P}_{s}$ vs. average SNR, for different values of $q, K, r$ (eavesdropper channel) and $m$ (legitimate channel). Parameter values are $\bar{\gamma}_{e}=0 \mathrm{~dB}$ and $R_{s}=0$ (strictly positive secrecy capacity).

components, has a negative impact on the detection probability compared to the balanced cases $(r=1$ or $q=1)$.

For the outage probability in interference-limited scenarios, we show how the OP performance improves when the LOS power is greater (greater $K$ ), when the spatial diversity increases (greater $L$ ) or when $q \rightarrow 1$. Once again, imbalance in the desired fading channel is a synonym of performance loss.

Finally, when analyzing the physical layer security, we showed that the eavesdropper fading conditions are dominant in the low-SNR regime. Conversely, in the high SNR regime the behavior is just the opposite, and the fading experienced by legitimate receiver determines the probability of achieving a secure transmission.

\section{APPENDiX A \\ PROOF OF LEMMA I}

Proof: Let $R^{2}$ denote the power envelope of the Beckmann distribution, i.e., $R^{2} \triangleq X^{2}+Y^{2}$ such that $X \sim$ $\mathcal{N}\left(\mu_{x}, \sigma_{x}^{2}\right)$ and $Y \sim \mathcal{N}\left(\mu_{y}, \sigma_{y}^{2}\right)$, being $X$ and $Y$ independent. 
Both $X^{2}$ and $Y^{2}$ follow a non-central chi-squared distribution, which PDF is given by [1, eq. (2.1-115)]

$$
f(z)=\frac{1}{\sqrt{2 \pi z} \sigma_{i}} \exp \left[\frac{-\left(z+\mu_{i}^{2}\right)}{2 \sigma_{i}^{2}}\right] \cosh \left(\frac{\mu_{i} \sqrt{z}}{\sigma_{i}^{2}}\right), z \geq 0,
$$

where the subindex $i$ denotes either $x$ or $y$.

Given that $R^{2 n}=\sum_{k=0}^{n}\left(\begin{array}{l}n \\ k\end{array}\right) X^{2 k} Y^{2(n-k)}$ and assuming the independence of $X$ and $Y$, the desired expected value is obtained by averaging over both distributions, considering that each one of them follows (35), yielding,

$$
\begin{aligned}
& E_{R^{2}}\left\{R^{2 n} e^{s R^{2}}\right\}=\sum_{k=0}^{n}\left(\begin{array}{l}
n \\
k
\end{array}\right) E\left\{X^{2 k} e^{s X^{2}}\right\} E\left\{Y^{2(n-k)} e^{s Y^{2}}\right\} \\
& =\frac{1}{2 \pi \sigma_{x} \sigma_{y}} \sum_{k=0}^{n}\left(\begin{array}{l}
n \\
k
\end{array}\right) \\
& \times \int_{0}^{\infty} y_{1}^{k-1 / 2} \exp \left[s y_{1}-\frac{y_{1}+\mu_{x}^{2}}{2 \sigma_{x}^{2}}\right] \cosh \left(\frac{\mu_{x} \sqrt{y_{1}}}{\sigma_{x}^{2}}\right) d y_{1} \\
& \times \int_{0}^{\infty} y_{2}^{n-k-1 / 2} \exp \left[s y_{2}-\frac{y_{2}+\mu_{y}^{2}}{2 \sigma_{y}^{2}}\right] \cosh \left(\frac{\mu_{y} \sqrt{y_{2}}}{\sigma_{y}^{2}}\right) d y_{2} .
\end{aligned}
$$

In order to solve these integrals it is convenient to calculate the Laplace transform

$$
\begin{aligned}
F(z) & =\mathfrak{L}\left\{x^{m-1 / 2} \cosh (a \sqrt{x}) ; z\right\} \\
& =\frac{1}{2} \int_{0}^{\infty} x^{m-1 / 2} e^{-z x}\left[e^{a \sqrt{x}}+e^{-a \sqrt{x}}\right] d x \\
& =\int_{0}^{\infty} y^{2 m} \exp \left[-z y^{2}+a y\right] d y \\
& +\int_{0}^{\infty} y^{2 m} \exp \left[-z y^{2}-a y\right] d y,
\end{aligned}
$$

where we have made the change of variables $y=\sqrt{x}$. Now, let us introduce [24, eq. (3.462.1)]

$$
\begin{aligned}
\int_{0}^{\infty} & y^{\nu-1} \exp \left[-\beta y^{2}-\alpha y\right] d y \\
& =(2 \beta)^{-\nu / 2} \Gamma(\nu) \exp \left[\frac{\alpha^{2}}{8 \beta}\right] D_{-\nu}\left(\frac{\alpha}{\sqrt{2 \beta}}\right) ; \\
& \Re(\nu)>0 \wedge \Re(\beta)>0,
\end{aligned}
$$

where $\Re(z)$ denotes the real part of complex number $z$, and $D_{\nu}(z)$ is the parabolic-cylinder function defined in [24, eq. (9.240)] as

$$
\begin{aligned}
D_{\nu}(z) & =2^{\nu / 2} e^{-z^{2} / 4} \sqrt{\pi}\left[\frac{1}{\Gamma\left(\frac{1-\nu}{2}\right)}{ }_{1} F_{1}\left(\frac{-\nu}{2} ; \frac{1}{2} ; \frac{z^{2}}{2}\right)\right. \\
& \left.-\frac{a \sqrt{2}}{\Gamma\left(\frac{-\nu}{2}\right)}{ }_{1} F_{1}\left(\frac{1-\nu}{2} ; \frac{3}{2} ; \frac{z^{2}}{2}\right)\right],
\end{aligned}
$$

where ${ }_{1} F_{1}(a ; b ; x)$ is the confluent hypergeometric function of the first kind as defined in [26, eq. (13.1.2)]. Then, we can write

$$
\begin{aligned}
F(z) & =(2 z)^{-m-1 / 2} \Gamma(2 m+1) \exp \left[\frac{a^{2}}{8 z}\right] \\
& \times\left[D_{-2 m-1}\left(\frac{-a}{\sqrt{2 z}}\right)+D_{-2 m-1}\left(\frac{a}{\sqrt{2 z}}\right)\right] ; \\
& \Re(m)>-1 / 2 \wedge \Re(z)>0 .
\end{aligned}
$$

Now, introducing (39) into (40) we obtain

$$
\begin{gathered}
F(z)=2^{-2 m} \sqrt{\pi} \frac{(2 m) !}{m !} z^{-m-1 / 2}{ }_{1} F_{1}\left(m+\frac{1}{2} ; \frac{1}{2} ; \frac{a^{2}}{4 z}\right), \\
\Re(m)>-1 / 2 \wedge \Re(z)>0 .
\end{gathered}
$$

Therefore, (36) becomes

$$
\begin{aligned}
E_{R^{2}} & \left\{R^{2 n} e^{s R^{2}}\right\}=\frac{1}{2 \pi \sigma_{x} \sigma_{y}} \exp \left[-\frac{\mu_{x}^{2}}{2 \sigma_{x}^{2}}-\frac{\mu_{y}^{2}}{2 \sigma_{y}^{2}}\right] \sum_{k=0}^{n}\left(\begin{array}{l}
n \\
k
\end{array}\right) \\
\times & \mathfrak{L}\left\{x^{k-1 / 2} \cosh \left(\frac{\mu_{x}}{\sigma_{x}^{2}} \sqrt{x}\right) ; \frac{1}{2 \sigma_{x}^{2}}-s\right\} \\
\times & \mathfrak{L}\left\{x^{n-k-1 / 2} \cosh \left(\frac{\mu_{y}}{\sigma_{y}^{2}} \sqrt{x}\right) ; \frac{1}{2 \sigma_{y}^{2}}-s\right\} .
\end{aligned}
$$

Therefore, considering (37) and (41), after some manipulations we obtain

$$
\begin{aligned}
& \phi_{R^{2}}^{(n)}(s)=\frac{1}{\sigma_{y} \sigma_{x} 2^{2 n+1}} \exp \left[-\frac{\mu_{x}^{2}}{2 \sigma_{x}^{2}}-\frac{\mu_{y}^{2}}{2 \sigma_{y}^{2}}\right] \sum_{k=0}^{n}\left(\begin{array}{l}
n \\
k
\end{array}\right) \\
& \quad \times \frac{(2 k) !(2(n-k)) !}{k !(n-k) !}\left(\frac{2 \sigma_{x}^{2}}{1-2 \sigma_{x}^{2} s}\right)^{k+\frac{1}{2}}\left(\frac{2 \sigma_{y}^{2}}{1-2 \sigma_{y}^{2} s}\right)^{n-k+\frac{1}{2}} \\
& \quad \times{ }_{1} F_{1}\left(k+\frac{1}{2} ; \frac{1}{2} ; \frac{\mu_{x}^{2}}{2 \sigma_{x}^{2}\left(1-2 \sigma_{x}^{2} s\right)}\right) \\
& \quad \times{ }_{1} F_{1}\left(n-k+\frac{1}{2} ; \frac{1}{2} ; \frac{\mu_{y}^{2}}{2 \sigma_{y}^{2}\left(1-2 \sigma_{y}^{2} s\right)}\right)
\end{aligned}
$$

Taking into account that, for the parameter values in (43), the confluent hypergeometric function can be expressed, using [38, eq. (07.20.03.0007.01) and (05.08.06.0006.01)], in terms of a finite combination of elementary functions as

$$
\begin{aligned}
& { }_{1} F_{1}(a ; a-n ; z)=\frac{(-1)^{n} e^{z}}{(1-a)_{n}} \\
& \quad \times \sum_{m=0}^{n} \frac{(-n)_{m}(a-n+m)_{n-m}}{m !}(-z)^{m}
\end{aligned}
$$

where $(a)_{n}$ denotes the Pochhammer symbol, and with the help of equalities

$$
\begin{gathered}
\left(\frac{1}{2}+m\right)_{k-m}=\frac{1}{2^{2(k-m)}} \frac{(2 k) !}{k !} \frac{m !}{(2 m) !}, \text { for } k \geqslant m, \\
(-k)_{m}=(-1)^{m} \frac{k !}{(k-m) !}, \text { for } k \geqslant m, \\
\left(\frac{1}{2}-k\right)_{k}=(-1)^{k} \frac{(2 k) !}{k ! 2^{2 k}}
\end{gathered}
$$


Table II

NUMBER OF TERMS REQUIRED TO OBTAIN A RELATIVE ERROR LOWER THAN $10^{-6}$ FOR DIFFERENT SETS OF PARAMETERS.

\begin{tabular}{c|c|c|c|c|c|c}
\hline \hline $\bar{\gamma}$ & $q$ & $K$ & $r$ & $\lambda$ & $u$ & Needed Terms \\
\hline \hline $0 d B$ & 0.5 & 2 & 1 & $0 d B$ & 5 & 7 \\
\hline$-20 d B$ & 0.5 & 2 & 1 & $0 d B$ & 5 & 4 \\
\hline $40 d B$ & 0.5 & 2 & 1 & $0 d B$ & 5 & 7 \\
\hline $0 d B$ & 0.1 & 2 & 1 & $0 d B$ & 5 & 7 \\
\hline $0 d B$ & 0.9 & 2 & 1 & $0 d B$ & 5 & 7 \\
\hline $0 d B$ & 0.5 & 0.2 & 1 & $0 d B$ & 5 & 7 \\
\hline $0 d B$ & 0.5 & 20 & 1 & $0 d B$ & 5 & 6 \\
\hline $0 d B$ & 0.5 & 2 & 0.1 & $0 d B$ & 5 & 7 \\
\hline $0 d B$ & 0.5 & 2 & 10 & $0 d B$ & 5 & 7 \\
\hline $0 d B$ & 0.5 & 2 & 1 & $-20 d B$ & 5 & 5 \\
\hline $0 d B$ & 0.5 & 2 & 1 & $20 d B$ & 5 & 17 \\
\hline $0 d B$ & 0.5 & 2 & 1 & $0 d B$ & 2 & 7 \\
\hline $0 d B$ & 0.5 & 2 & 1 & $0 d B$ & 15 & 2 \\
\hline$-20 d B$ & 0.1 & 0.2 & 0.1 & $-20 d B$ & 2 & 4 \\
\hline $40 d B$ & 0.9 & 20 & 10 & $20 d B$ & 15 & 71 \\
\hline \hline
\end{tabular}

which can be easily demonstrated by mathematical induction, after some manipulation we can write

$$
\begin{aligned}
& \phi_{R^{2}}^{(n)}(s)=\frac{1}{\sqrt{\left(1-2 \sigma_{x}^{2} s\right)\left(1-2 \sigma_{y}^{2} s\right)}} \\
& \quad \times \exp \left(\frac{\mu_{x}^{2} s}{1-2 \sigma_{x}^{2} s}+\frac{\mu_{y}^{2} s}{1-2 \sigma_{x}^{2} s}\right) \\
& \quad \times \frac{n !}{2^{2 n}} \sum_{k=0}^{n} \frac{(2 k) !(2(n-k)) !}{k !(n-k) !} \\
& \quad \times\left(\frac{2 \sigma_{x}^{2}}{1-2 \sigma_{x}^{2} s}\right)^{k}\left(\frac{2 \sigma_{y}^{2}}{1-2 \sigma_{y}^{2} s}\right)^{n-k} \\
& \times\left(\sum_{m=0}^{k} \frac{\left(4 z_{x}\right)^{m}}{(k-m) !(2 m) !}\right)\left(\sum_{m=0}^{n-k} \frac{\left(4 z_{y}\right)^{m}}{(n-k-m) !(2 m) !}\right) .
\end{aligned}
$$

where $z_{x}=\frac{\mu_{x}^{2}}{2 \sigma_{x}^{2}\left(1-2 \sigma_{x}^{2} s\right)}, z_{y}=\frac{\mu_{y}^{2}}{2 \sigma_{y}^{2}\left(1-2 \sigma_{y}^{2} s\right)}$. 00 Considering now that $\gamma=R^{2} E_{s} / N_{0}=\left(X^{2}+Y^{2}\right) E_{s} / N_{0}$, the statistics of $\gamma$ will be the same as the ones for $R^{2}$ by just scaling parameters $\mu_{x}, \mu_{y}, \sigma_{x}$ and $\sigma_{y}$ by $\sqrt{E_{s} / N_{0}}$. Therefore, from (48) and using the parameters defined in Section II, after some algebraic manipulation, (6) is obtained.

\section{APPENDIX B}

Convergence of $\overline{P_{m}}$

The Marcum- $Q$ function in (13) can be conveniently reexpressed as

$$
Q_{u}(\sqrt{2 \gamma}, \sqrt{\lambda})=1-e^{-\gamma} \sum_{n=0}^{\infty} \frac{\gamma^{n}}{n !} \frac{g(u+n, \lambda / 2)}{\Gamma(u+n)},
$$

where we used the definition of the lower incomplete gamma function $g(n, x)=\Gamma(n)-\Gamma(n, x)$.
From (49), (12) and (14), the missed detection probability can be written as

$$
\begin{aligned}
\overline{P_{m}} & =\sum_{n=0}^{\infty} \frac{1}{n !} \frac{g(u+n, \lambda / 2)}{\Gamma(u+n)} \phi_{\gamma}^{(n)}(-1) \\
& =\sum_{n=0}^{\infty} \frac{1}{n !}\left[1-e^{-\lambda / 2} \sum_{k=0}^{u+n-1} \frac{1}{k !}\left(\frac{\lambda}{2}\right)^{k}\right] \phi_{\gamma}^{(n)}(-1) .
\end{aligned}
$$

In order to analyze the convergence of (50), we can numerically evaluate the convergence speed for different sets of parameters as shown in Table II. We see that the series converges very fast except for high values of $\lambda$, especially in the high-SNR regime. However, the value of the threshold parameter $\lambda$ is seldom larger a few $d B$. Even in such cases, we must note that evaluating the first hundreds of terms is rather fast, as we are actually computing elementary functions (i.e., powers and exponentials).

\section{ACKNOWLEDGMENT}

The work of Juan P. Peña-Martín and Juan M. Romero-Jerez was supported by the Spanish Government-FEDER public Project No. TEC2013-42711-R. The work of F.J. LopezMartinez was funded by Junta de Andalucia (P11-TIC-7109) and the Spanish Government-FEDER (TEC2013-44442-P).

\section{REFERENCES}

[1] J. G. Proakis, Digital Communications, 4th ed. McGraw Hill, 2001

[2] P. Beckmann, "Statistical distribution of the amplitude and phase of a multiply scattered field," JOURNAL OF RESEARCH of the National Bureau of Standards-D. Radio Propagation, vol. 66D, no. 3, pp. 231240, 1962.

[3] — "Rayleigh distribution and its generalizations," RADIO SCIENCE Journal of Research NBS/USNC-URSI, vol. 66D, no. 3, pp. 231-240, 1964.

[4] V. A. Aalo, G. P. Efthymoglou, and C. Chayawan, "On the envelope and phase distributions for correlated gaussian quadratures," IEEE Commun. Lett., vol. 11, no. 12, pp. 985-987, Dec. 2007.

[5] P. Beckmann and A. Spizzichino, The Scattering of Electromagnetic Waves from Rough Surfaces, 2nd ed. Boston, MA: Artech House, 1987.

[6] S. O. Rice, "Mathematical analysis of random noise," The Bell System Technical Journal, vol. 23, no. 3, pp. 282-332, Jul. 1944.

[7] R. S. Hoyt, "Probability functions for the modulus and angle of the normal complex variate," The Bell System Technical Journal, vol. 26, no. 4, pp. 318-359, 1947.

[8] M. Yacoub, "The $\kappa-\mu$ distribution and the $\eta-\mu$ distribution," IEEE Antennas Propag. Mag., vol. 49, no. 1, pp. 68-81, Feb. 2007.

[9] J. Paris, "Statistical characterization of $\kappa-\mu$ shadowed fading," IEEE Trans. Veh. Technol., vol. 63, no. 2, pp. 518-526, Feb. 2014.

[10] Y. Xie and Y. Fang, "A general statistical channel model for mobile satellite systems," IEEE Trans. Veh. Technol., vol. 49, no. 3, pp. 744752, May 2000

[11] H. T. Dougherty, A survey of microwave fading mechanisms remedies and applications. Wave Propagation Laboratories, 1968, vol. 52.

[12] N. Youssef, C.-X. Wang, M. Patzold, I. Jaafar, and S. Tabbane, "On the statistical properties of generalized Rice multipath fading channels," in Vehicular Technology Conference, VTC 2004-Spring. 2004 IEEE 59th, vol. 1, May 2004, pp. 162-165.

[13] P. Dharmawansa, N. Rajatheva, and C. Tellambura, "Envelope and phase distribution of two correlated gaussian variables," IEEE Trans. Commun., vol. 57, no. 4, pp. 915-921, Apr. 2009.

[14] G. A. Ropokis, A. A. Rontogiannis, P. T. Mathiopoulos, and K. Berberidis, "An Exact Performance Analysis of MRC-OSTBC over Generalized Fading Channels," IEEE Trans. Commun., vol. 58, no. 9, pp. 2486-2492, Sep. 2010.

[15] W. Dahech, N. Hajri, N. Youssef, T. Kawabata, and M. Pätzold, "Outage statistics for Beckmann fading channels in non-isotropic scattering 
environments," in 21st Asia-Pacific Conference on Communications (APCC) 2015, Oct. 2015, pp. 164-168.

[16] B. Zhu, J. Cheng, N. Al-Dhahir, and L. Wu, "Asymptotic analysis and tight performance bounds of diversity receptions over beckmann fading channels with arbitrary correlation," IEEE Trans. Commun., vol. 64, no. 5, pp. 2220-2234, May 2016.

[17] M. K. Simon and M.-S. Alouini, Digital Communications over Fading Channels, 2nd ed. John Wiley \& Sons, Inc., 2005.

[18] M. D. Yacoub, G. Fraidenraich, H. B. Tercius, and F. C. Martins, "The symmetrical $\eta-\kappa$ distribution: a general fading distribution," IEEE Trans. Broadcast., vol. 51, no. 4, pp. 504-511, Dec. 2005.

[19] —, "The asymmetrical $\eta-\kappa$ distribution," Journal of Communication and Information Systems, vol. 20, no. 3, pp. 182-187, 2005.

[20] H. Urkowitz, "Energy detection of unknown deterministic signals," Proceedings of the IEEE, vol. 55, no. 4, pp. 523-531, Apr. 1967.

[21] A. Annamalai, O. Olabiyi, S. Alam, O. Odejide, and D. Vaman, "Unified analysis of energy detection of unknown signals over generalized fading channels," in Wireless Communications and Mobile Computing Conference (IWCMC), 2011 7th International, Jul. 2011, pp. 636-641.

[22] O. Olabiyi, S. Alam, O. Odejide, and A. Annamalai, "Efficient evaluation of area under the ROC curve of energy detectors over fading channels," in Proc.ACM/IEEE MSWiM'11. NY, USA: ACM, 2011, pp. 261-264.

[23] F. Digham, M.-S. Alouini, and M. K. Simon, "On the energy detection of unknown signals over fading channels," in Proc. IEEE International Conference on Communication. ICC '03., vol. 5, May 2003, pp. 35753579.

[24] I. S. Gradshteyn and I. M. Ryzhik, Table of Integrals, Series and Products, 6th ed. Academic Press, 2000.

[25] S. Atapattu, C. Tellambura, and H. Jiang, "Analysis of area under the ROC curve of energy detection," IEEE Trans. Wireless Commun., vol. 9, no. 3, pp. 1216-1225, Mar. 2010.

[26] M. Abramowitz and I. A. Stegun, Handbook of Mathematical Functions with Formulas, Graphs, and Mathematical Tables, 10th ed. U.S. Department of Commerce - N.B.S., Dec. 1972.

[27] P. C. Sofotasios, M. K. Fikadu, K. Ho-Van, M. Valkama, and G. K Karagiannidis, "The area under a receiver operating characteristic curve over enriched multipath fading conditions," in 2014 IEEE Global Communications Conference, Dec. 2014, pp. 3490-3495.

[28] A. Bagheri, P. C. Sofotasios, T. A. Tsiftsis, A. Shahzadi, and M. Valkama, "AUC study of energy detection based spectrum sensing over $\eta-\mu$ and $\alpha-\mu$ fading channels," in 2015 IEEE International Conference on Communications (ICC), June 2015, pp. 1410-1415.

[29] — , "Spectrum sensing in generalized multipath fading conditions using square-law combining," in 2015 IEEE International Conference on Communications (ICC), Jun. 2015, pp. 7528-7533.

[30] A. Bagheri, P. C. Sofotasios, T. A. Tsiftsis, A. Shahzadi, S. Freear, and M. Valkama, "Area under ROC curve of energy detection over generalized fading channels," in 2015 IEEE 26th Annual International Symposium on Personal, Indoor, and Mobile Radio Communications (PIMRC), Aug 2015, pp. 656-661.

[31] J. P. Peña Martín and J. M. Romero-Jerez, "Outage probability with MRC in presence of multiple interferers under Rayleigh fading channels," IEE Electron. Lett., vol. 40, no. 14, pp. 888-889, Jul. 2004.

[32] J. M. Romero-Jerez and A. J. Goldsmith, "Exact error rates of MRC with transmit antenna selection in non-identically distributed Nakagami fading channels," in Proc. IEEE Global Communications Conference (GLOBECOM'08), New Orleans - U.S.A., Dec. 2008.

[33] A. Shah and A. Haimovich, "Performance analysis of maximal ratio combining and comparison with optimum combining for mobile radio communications with cochannel interference," IEEE Trans. Veh. Technol., vol. 49, pp. 1454-1463, Jul. 2000.

[34] J. M. Romero-Jerez and A. J. Goldsmith, "Receive antenna array strategies in fading and interference: an outage probability comparison," IEEE Trans. Wireless Commun., vol. 7, no. 3, pp. 920-932, 2008.

[35] S. Leung-Yan-Cheong and M. Hellman, "The Gaussian wire-tap channel," IEEE Trans. Inf. Theory, vol. 24, no. 4, pp. 451-456, Jul. 1978.

[36] J. Barros and M. R. D. Rodrigues, "Secrecy capacity of wireless channels," in 2006 IEEE International Symposium on Information Theory, Jul. 2006, pp. 356-360.

[37] J. M. Romero-Jerez, G. Gomez, and F. J. Lopez-Martinez, "On the outage probability of secrecy capacity in arbitrarily-distributed fading channels," in European Wireless 2015; 21th European Wireless Conference; Proceedings of, May 2015, pp. 1-6.

[38] "The wolfram function site," Wolfram Research, Inc., 2016. [Online]. Available: http://functions.wolfram.com/

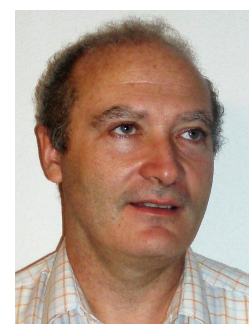

Juan P. Peña-Martín received the M.Sc. degree in Physics from the University of Granada, Spain, in 1986 and the Ph.D. degree from the University of Málaga, Spain, in 2009.

Currently, he is Associate Professor in the Electronic Technology Department of the University of Málaga, Spain. He started his cooperation with the University of Málaga in 1988, as a part-time lecturer. Since 1986 until 1992, he worked as a hardware engineer in the R\&D Department of Fujitsu España, SA. and, for one year, in the Kawasaki Laboratories of Fujitsu Limited. Since 1992 until 2003, he worked in AT4-Wireless where he held various positions. Since 2003 until 2007, he was a freelance consultant for testing laboratories.

Nowadays, His research interests are in the areas of wireless communications performance analysis, multipath fading, wireless channel modeling, diversity systems and MIMO performances.

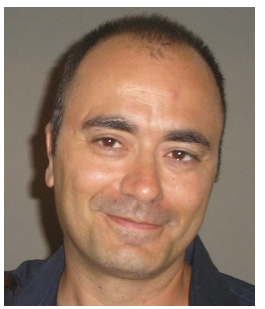

Juan M. Romero-Jerez has M.Sc. degrees both in Telecommunication Engineering and in Mathematics from the University of Málaga, Spain, where he also obtained a Ph.D. degree in Telecommunication Engineering in 2001. In 1996 he joined the Electronic Technology Department at the University of Málaga, where he is an Associate Professor since 2002. He was a Visiting Associate Professor in the Electrical Engineering Department at Stanford University from September 2005 to February 2006, and also from September 2007 to February 2008 and from January 2016 to February 2016. He has participated in several research projects in the areas of packet radio transmission, multiple antennas, interference management and cellular networks.

His current research interest is in the area of wireless communications and, more specifically: wireless communications performance analysis, multipath fading, wireless channel modeling, diversity systems, smart antennas, MIMO performance and interference management.

$\mathrm{He}$ is a Senior Member of the IEEE and also an Editor of the IEEE Transactions on Wireless Communications.

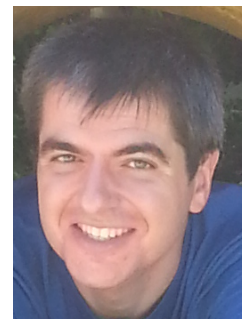

F. Javier Lopez-Martinez received the M.Sc. and $\mathrm{Ph} . \mathrm{D}$. degrees in telecommunication engineering from the University of Málaga, Spain, in 2005 and 2010, respectively. He joined the Communication Engineering Department with the University of Málaga in 2005, as an Associate Researcher. In 2010, he stayed for three months as a Visitor Researcher with University College London. He was a Marie Curie Post-Doctoral Fellow with the Wireless Systems Lab, Stanford University, from 2012 to 2014, and with the University of Málaga from 2014 to 2015. Since 2015, he has been an Assistant Professor with the Communication Engineering Department, University of Málaga. His research interests span a diverse set of topics in the wide areas of communication theory and wireless communications: stochastic processes, wireless channel modeling, random matrix theory, physical layer security, massive MIMO, and mmWave for 5G. He has received several research awards, including the Best Paper Award in the Communication Theory Symposium at IEEE Globecom 2013, the IEEE Communications Letters Exemplary Reviewer certificate in 2014, and the IEEE Transactions on Communications Exemplary Reviewer certificate in 2015 and 2017. He is an Editor of the IEEE Transactions on Communications, in the area of Wireless Communications. 